\title{
Analysis of Misdiagnosis Cases for Acute Abdomen Cardiovascular Disease
}

\author{
Huaijun Du* \\ Kuo Cheng Yi Center Hospital of Huining County, Baiyin, Gansu 730726, China
}

\begin{abstract}
With the continuous improvement of the grassroots medical reform, the basic medical care for human being arose in order to avoid problems. Complicated cases faced by the grassroots doctors in treating patients was largely due several factors namely, the hospital equipment, coupled with most doctors have not yet been fully trained as general practitioner, knowledge limitations, less clinical experiences especially in terms of emergency patients' cases, treatment was not given in time, not having accurate diagnosis, and mistreatment. Facing with the current medical condition, therefore it is the main purpose of this study to deal with the phenomenon of the patients that were unable to be treated in time. The study involved the cases of cardiovascular diseases which had been misdiagnosed as acute abdomen to exemplify China's grassroots misdiagnosis phenomenon, by providing summary from the analysis and expounding on how to avoid misdiagnosis phenomenon at the grass-roots level.
\end{abstract}

\author{
KEYWORDS \\ Acute abdomen \\ Cardiovascular \\ Misdiagnosis
}

\section{Introduction}

According to the national medical school statistics, the average number of patients' visit per year was 1000 cases while the number of patients in the primary treatment was also more than 100 cases. This phenomenon was due to the misdiagnosis of patients which was mostly conducted not in time with the number of patients was more than 60 . Moreover, it was also due to the primary care of medical equipment and priority went to medical Ninjutsu. By doing so, judgment to the patient's condition could be made and able to respond them promptly as the daily visits of patients inclining. The diseases were different among patients, in which, the diagnosis judgement was mostly inaccurate for each patient. This would result in misdiagnosis phenomenon especially when it comes to urgent referral treatment of misdiagnosis phenomenon whereby it could

Copyright $\odot 2015$ Huaijun Du

doi: $10.18686 /$ aem.v4i4.9

Received: September 12, 2015; Accepted: November 16, 2015; Published online: December 30, 2015

This is an open-access article distributed under the terms of the Creative Commons Attribution Unported License (http://creativecommons.org/ licenses/by-nc/4.0/), which permits unrestricted use, distribution, and reproduction in any medium, provided the original work is properly cited.

${ }^{*}$ Corresponding author: Kuo Cheng Yi Center Hospital of Huining County, Baiyin, Gansu 730726, China. E-mail: li_zhangyu1981@sina. com lead to much serious cases. The clinical diagnosis was conducted and treatment data of realistic experience of three cases of both emergency and referral clinics was analyzed for misdiagnosis and mistreatment of cardiovascular disease in patients. The summary was as follows.

\section{General information and methods 2.1. Case one}

Superior mesenteric artery embolism was misdiagnosed as acute gastroenteritis, acute myocardial infarction, and acute appendicitis. 75 years old male patient who was having "paroxysmal abdominal pain with mild diarrhea for two days" came to our hospital as outpatient, however, admitted with acute gastroenteritis. Patient private prosecution of previously healthy, before the onset of the disease can also be shepherd. After the onset of a clinic administration of antidiarrheal drugs, eventually diarrhea stopped (specific drug name ominous). Admission examination body was: T: $37.2^{\circ} \mathrm{C}, \mathrm{P}: 160$ beats $/ \mathrm{min}, \mathrm{R}: 20$ breaths $/ \mathrm{min}$, Bp: 140/85 mmHg. Patient's mental status was relatively low most of the time except during episodes of paroxysmal abdominal pain with short-term pain expression. Auscultation of heart rate and strength was unheard, the pathological heart murmurs, evenly-breathed, with no abnormal lung auscultation. Abdominal examination: body turned slim, scaphoid abdomen, liver was not lineal, total abdominal of untouched abnormal mass, upper abdomen 
showed mild tenderness and painful abdominal without bouncing. Auxiliary examination: electrocardiogram showing atrial fibrillation, ventricular rate of 160 times/ points, peritoneal dialysis hinted mild intestinal flatulence, blood and urine showed no abnormality in routine, and stool routine was not found. After treatment: patients have rapid atrial fibrillation, however, doctor examined patients for cardiovascular symptoms such as shortness of breath and heartbeat feeling of discomfort, and other abdominal examination. Once considered as "acute gastroenteritis", acid suppression was given due to occurrence of spasmolysis. After symptomatic treatment for atrial fibrillation, no symptoms found yet. The patients supine abdominal pain symptoms disappeared once the symptoms of abdominal pain eased at the same night. The patient's family informed that the patient often urinates at night every day. After the emergence of a more severe abdominal pain, the patient went for fast checking of myocardial enzymes examination in order to detect the presence of creatine kinase, creatine kinase isoenzyme, lactate dehydrogenase, and alphahydroxybutyrate dehydrogenase in different degree. $\mathrm{He}$ was then suspected with acute myocardial infarction and return to the hospital for treatment.

Analysis of the causes of misdiagnosis. This case can be described as one type of misdiagnosis of patients that lead to death. Doctors at all levels should drew a lesson. In this case, we should review the relevant knowledge of atrial fibrillation and mesenteric artery embolism. The elderly patients tend to have atrial fibrillation (AF) and their ventricular rate was fast, as well as those without heart-related symptoms that persist for a long time. Their body could tolerate with that kind of situation whereas it was viceversa for antecedent AF patients who were not undergo examination and anticoagulation therapy. One of the most common complications of atrial fibrillation is embolism, which occurs in the arteries of the brain, lung, limbs, and the superior mesenteric artery. Embolism that occurs at lower abdomen has the probability of developing mesenteric and the other abdominal disease symptoms. Embolism was difficult to detect under inconvenient and unreliable examination method. Therefore, rate of misdiagnosis for the disease was rather high. The clinical characteristics of mesenteric arterial embolization ware that the disease often occurs in patients with atrial fibrillation. The onset of the symptoms was acute, and the signs were inconsistent with severe abdominal pain. Gastric emptying was the clinical characteristics of mesenteric arterial embolization. At the beginning of the disease, abdominal pain was not severe in the patient and often there was a relieved period. The abdominal pain event faced by the patients was different for each individual and could be associated with the degree of embolism, location and posture.

\subsection{Case two}

Acute myocardial infarction was usually misdiagnosed as acute cholecystitis. Male patient of 65 years old claimed of having acute upper abdominal pain for three hours. The patient possesses more than ten year's history of hypertension and was treated with irregular oral compound of antihypertensive tablets which is known as Betaloc. During admission, the blood pressure was $160 / 100 \mathrm{mmHg}$, with diastolic heart rate was 75 . Abdomen body examination: The abdomen was soft and the tenderness was obvious in upper abdominal indicating a positive Murphy's sign. The lower abdomen did not show any tenderness. Detection of myocardial enzymes i.e., creatine kinase, lactate dehydrogenase, alpha-hydroxybutyrate dehydrogenase was low. Two hours after admission, the right bundle branch block showed mild elevation of the first two V1-V3 ST segment. Nitroglycerin of $10 \mathrm{mg}$ was given thrice daily after the examination of ECG was conducted. The right bundle branch block disappeared suggesting that the treatment worked out. ST segment and the first two showed no significant changes in patients with abdominal pain. B exceeds clew: enlargement of the gallbladder $(12 \mathrm{~cm} \times 4 \mathrm{~cm})$, after the patient had his dinner. He had a positive Murphy's sign and the case was diagnosed as acute cholecystitis. The patient was given anti-inflammatory antispasmodic and symptomatic treatment. The next morning, the abdominal pain was still alleviated, and re-checked using electrocardiogram (ECG) and underwent myocardial enzyme detection. In ECG, there was no significant changes, however, the myocardial enzymes increased gradually especially for the creatine kinase isoenzyme. The patient was confirmed with myocardial infarction and transferred to higher hospital for further treatment, which has been re-confirmed of having myocardial infarction by coronary angiography in the higher level hospitals.

The cause of misdiagnosis analysis: The diagnosis of the patients was merely due to the interference factors that the authors need to learn more on it. Firstly, appearance of clinical abdominal symptoms in acute myocardial infarction were common. Therefore, elderly patients with abdominal pain should not neglect the cardiovascular examination which is not just necessarily relying on the abdomen examination. Previous medical history should be taken into high consideration especially the one that related to patients' cardiovascular and gallbladder examinations so that the diagnosis of would not astray. Secondly, electrocardiogram (ECG) in the diagnosis of acute myocardial infarction (MI) was the only reference used in clinical interpretation. Indeed, there were several patients diagnosed for myocardial infarction, but the ECG displayed completely normal and different result, which was known as the ST elevation myocardial infarction. Less experiences and lack of knowledge have been another factors to overcome since electrocardiogram (ECG)-based examination for myocardial infarction often delayed the diagnosis and treatment of disease. Dynamic changes of electrocardiogram (ECG) is often overlooked as this is valuable for the 
diagnosis of myocardial infarction which was based on the disappearance of the right bundle branch block after the consumption of the nitroglycerin. It was strongly suggested that the existence of the cardiac lesion was not taken seriously by our staff, therefore acute myocardial infarction (AMI) in the patient could not rule out at several interval times. Detection for myocardial enzymes had to be conducted a few times or more in order to avoid misdiagnosis of the patients with acute myocardial infarction disease.

\subsection{Case three}

Diagnosis of ureteral calculi with aortic dissection. Male patient of 50 years old was admitted due to "in abdominal and right lumbar back pain one hour visit". He experienced sweating, pale and irritability, together with groaning right after admission. At the time of admission, the heart rate was 90 beats $/ \mathrm{min}$, with the blood pressure of $160 / 90 \mathrm{mmHg}$. The patient had hypertension history for more than five years under private prosecution. Physical examination: obvious abdominal tenderness was seen without painful bouncing, right kidney area percussion pain was positive, urgent B-mode ultrasonography and urine routine check were carried out. The ultrasound for the diagnosis of ureteral calculi showed mild right kidney effusion, with urine routine test indicated positive occult blood. One hour after feeling pain, the patient was given with pethidine of $100 \mathrm{mg}$ intramuscular but still not showing any relieves. He was transferred to a secondary hospital in order to receive quasi ESWL therapy and underwent ureteral calculi diagnosis for aortic dissection. "However, from the onset to death, which was before and after the insufficient of 6 hours treatment, the patient died on the way to the tertiary hospital during transferal process from the secondary hospital.

Cause analysis: Patients having acute onset and rapid progression was defined as critical. The grassroot doctors had limited preconceived at the time of diagnosis. The auxiliary examination during the admission added to the doctor's confidence, in which, it lead to the misdiagnosis of the patient. Grassroot doctors thought of the concept of "afraid not to know, and they were afraid the unexpected". Despite the low incidence of clinical aortic dissection, grassroot doctors should always think of the possibility of the occurrence of aortic dissection in the hypertension patients that encountered severe abdominal pain since at times, patient appeared to be right for their speculation on hydronephrosis and hematuria, which is related to abdominal aorta and renal artery involvement.

\section{Discussion}

The above-mentioned cases were from the author's own experiences pertaining to misdiagnosis of acute abdomen symptoms in cardiovascular disease patients. One of the major causes of the misdiagnosis was due to the basic or minimal level of inspection on the equipment even though it was simple and limited. The clinical manifestations of disease were not typical or native with other objective reasons. However, the doctor's subjective reasons could not be ignored $[1,2]$. The foundation or theory pertaining to the disease was shallow, which is narrowing down the diagnosis process. The patient's medical history, symptoms, physical and auxiliary examinations were found to have problems with the analysis system in order to sift the true from the false. The medical personnel should make full use of the equipment and suggest for some valuable positive advice on that. Lesson must be learnt from the mistakes and constantly learning in order to enhance the sense of responsibility. Besides that, doctors could not be complacent, careless, and showing their doubt during consultation [3]. By following these methods, doctors are able to avoid misdiagnosis and error in order to minimize the pain of patients with the concept of "Entrusted health and life". One of the major clinicians' responsibilities is to have slight negligence that may lead to a big mistake which may reduce the mortality rate in the clinicians' hands and increase the quality of human life. By analyzing the three cases of cardiovascular diseases that had been misdiagnosed as acute abdomen, it was able to sum up that the grassroots medical misdiagnosis phenomenon can be improved by implementing the following aspects. Firstly, by inquiring the details of the medical history of patients as this can be the benchmark to treat the patients' disease due to the upcoming number of patients during diagnosis, and the initial manifestation of the disease was not obvious to be deduced, in which, resulted in the phenomenon of the primary consciousness. Secondly, by upgrading the basic knowledge or training for the basic medical treatment for the doctors. The needs for primary physicians to handle basic medical equipment for treatment of the patients was situation-based and they should summarized their findings based on their experiences, so that patients can be diagnosed and treated correctly $[4,5]$. Thirdly, by strengthening the communication with the local superior hospital. Due to the limited information exchange and slower transmission of the process, the primary physicians did not have enough time to understand the current disease situation and the virus that caused clinical symptoms. Therefore, occurrence of misdiagnosis was easily found in the process of medical treatment of the patients. The primary doctors should have communicated with the hospital in order to exchange the current information on the epidemic disease prevention and treatment, besides sharing the clinical and diagnostic experiences which apparently help in reducing the rate of misdiagnosis.

\section{References}

1. Zhao RS, Liao ZC, Diao WQ, Xu ZH, Chen H. With acute abdomen for the performance of colon cancer clinical diagnosis and treatment study. Chinese and Western Medical Treatment. 2013;07:6-7. 
2. Shi CF. Analysis of colon cancer complicated with acute abdomen misdiagnosed (22 cases report). Medical Theory and Practice. 2013;20:2706-2707.

3. $\mathrm{Hu} \mathrm{JH}$. Acute abdomen as colon cancer diagnosis and treatment performance. Chinese Med. 2012;06:972-973.

4. Tang CY, Zhi SM, Jiang XX, Huang JF. Emergency diagnosis and treatment of acute abdomen patients' clinical misdiagnosis analysis. Jilin Medical. 2013;19(01)3863-3864.

5. Qin QT, Zhang DH. With acute abdomen as the first symptom of acute myocardial infarction (AMI) misdiagnosis analysis. China Clinical Journal of Thoracic and Cardiovascular Surgery. 1996;04(01):33-35. 